\title{
Asthma: Hospitalization Trends and Predictors of In-Hospital Mortality and Hospitalization Costs in the USA (2001-2010)
}

\author{
Bani Preet Kaur ${ }^{a}$ Sopan Lahewala ${ }^{b}$ Shilpkumar Arora ${ }^{c}$ Kanishk Agnihotri ${ }^{a}$ \\ Sidakpal S. Panaich ${ }^{a}$ Elizabeth Secord $^{a}$ Diane Levine $^{a}$ \\ ${ }^{a}$ Detroit Medical Center, Wayne State University, Detroit, Mich., b Jersey City Medical Center, Jersey City, N.J., and \\ 'Mount Sinai St Luke's Roosevelt Hospital, New York, N.Y., USA
}

\section{Key Words}

Asthma - Length of stay · Cost of hospitalization .

In-hospital mortality

\begin{abstract}
Background: In the last decade, the proportion of people with asthma in the USA grew by nearly $15 \%$, with 479,300 hospitalizations and 1.9 million emergency department visits in 2009 alone. The primary objective of our study was to evaluate in-hospital outcomes in patients admitted with asthma exacerbation in terms of mortality, length of stay (LOS) and hospitalization costs. Methods: We queried the HCUP's Nationwide Inpatient Sample (NIS) between 2001 and 2010 using the ICD9-CM diagnosis code 493 for asthma ( $n=760,418$ patients). The NIS represents $20 \%$ of all hospitals in the USA. Multivariate logistic regression analysis was used to evaluate predictors of in-hospital mortality. LOS and hospitalization costs were also analyzed. Results: The overall LOS was 3.9 days and as high as 8.3 days in patients requiring mechanical ventilation. LOS has decreased in recent years, though it continues to be higher than in 2001. The hospitalization cost increased steadily over the study period. The overall in-hospital mortality was $1 \%$ and as high as $9.8 \%$ in patients requiring mechanical ventilation. Multivar-
\end{abstract}

iate predictors of longer LOS, higher hospitalization costs and in-hospital mortality included increasing age and hospitalizations during the winter months. Private insurance was predictive of lower hospitalization costs and LOS as well as lower in-hospital mortality. Conclusion: Asthma continues to account for significant in-hospital mortality and resource utilization, especially in mechanically ventilated patients. Age, admissions during winter months and the type of insurance are independent predictors of in-hospital outcomes.

(c) 2015 S. Karger AG, Basel

\section{Introduction}

Asthma is a highly prevalent respiratory illness [1] that accounts for more than 15 million physician office and hospital outpatient department visits, and nearly 2 million visits to the emergency department each year. As per data from the Centers for Disease Control and Prevention's (CDC) National Asthma Control Program, the proportion of people with asthma in the USA grew by

B.P.K., S.L. and S.A. contributed equally to this article.

Correspondence to: Dr. Bani Preet Kau

Detroit Medical Center, Wayne State University

4201 St. Antoine Street, Suite 2E

Detroit, MI 48201 (USA)

E-Mail bkaur@med.wayne.edu 
nearly $15 \%$ in the last decade. In 2009 , there were an estimated 24.6 million patients with asthma, resulting in 479,300 hospitalizations [2, 3]. Although most of the asthma patients are managed in the outpatient setting, severe exacerbation requires hospitalization and even intubation/mechanical ventilation. This further accounts for the significant morbidity, mortality and health care expenditures. Despite only $20 \%$ of asthmatics requiring hospitalization, these exacerbations along with emergency visits account for $80 \%$ of direct costs as per previous reports [4]. The primary objective of our study was to evaluate in-hospital outcomes in patients admitted with asthma exacerbation in terms of mortality, length of stay (LOS) and hospitalization costs.

\section{Methods}

The study cohort was derived from the Healthcare Cost and Utilization Project's (HCUP) Nationwide Inpatient Sample (NIS) database between 2001 and 2010, sponsored by the Agency for Healthcare Research and Quality (AHRQ). The NIS is the largest publicly available all-payer inpatient care database in the USA, including data on approximately 7-8 million discharges per year, and is a stratified sample designed to approximate a $20 \%$ sample of the US community (nonfederal, short-term, general and specialty) hospitals. National estimates are produced using sampling weights provided by the sponsor [5]. Weighted, it estimates more than 36 million hospitalizations nationally. The details regarding the NIS data have been previously published [6]. Annual data quality assessments of the NIS are performed, which guarantee the internal validity of the database. Furthermore, comparisons against the following data sources strengthen the external validity of the NIS: the American Hospital Association Annual Survey Database, the National Hospital Discharge Survey from the National Center for Health Statistics, and the MedPAR inpatient data from the Centers for Medicare and Medicaid Services [7, 8].

We queried the NIS database in 2014 for the period from 2001 to 2010 using the International Classification of Diseases, 9th Revision, Clinical Modification (ICD-9-CM). Patients with a primary diagnosis of asthma (ICD-9-CM code: 493) were included in the study $(\mathrm{n}=760,418$ patients, weighted $\mathrm{n}=3,743,613)$. Patients who died over the study period and those with missing data for sex or admission type were excluded.

NIS variables were used to identify patient demographic characteristics, including age, gender and race (table 1). The total duration of hospital stay in days was estimated for patients, after excluding those who died in the hospital, using the information on LOS provided in the NIS dataset. The NIS dataset includes all the patients admitted under observational or inpatient status into participating hospitals. The HCUP's NIS contains data on total charges for each hospital in the database, which represents the amount that hospitals billed for services. In order to calculate estimated hospitalization cost, the NIS data were merged with costto-charge ratios (CCR) available from HCUP. Using the merged data elements from the CCR files and the total charges reported in the NIS database, we converted the hospital total charge data to cost estimates by simply multiplying the total charges with the appropriate CCR. These costs are in essence standardized and can be measured across hospitals and are used in the remainder of this paper. The adjusted cost for each year was calculated in terms of the 2010 cost after adjusting for inflation according to the latest consumer price index data released by the US government on January $16,2013[9]$.

Stata IC 11.0 (Stata-Corp., College Station, Tex., USA) and SAS 9.3 (SAS Institute Inc., Cary, N.C., USA) were utilized for the analyses. Weighted values of patient level observations were generated to produce a nationally representative estimate of the entire US population of hospitalized patients. Differences between categorical variables were tested using the $\chi^{2}$ test and differences between continuous variables were tested using the Student $\mathrm{t}$ test. $\mathrm{p}<0.05$ was considered significant.

Multivariate logistic regression models were used for categorical dependent variables like in-hospital mortality, while multivariate linear regression models were used for continuous dependent variables such as cost of hospitalization and LOS. Variables with $>10 \%$ missing data were not included in the multivariate models. In all multivariate models, we included hospital-level variables like hospital region (Northeast, South, Midwest with West as the reference), teaching versus nonteaching hospital and patient level variables like age, sex, admission over the weekend and primary payer (with Medicare/Medicaid considered as the reference). All interactions were thoroughly tested. Multicollinearity, defined as a perfect linear relationship or very high correlation between two or more predictor (independent) variables, was assessed using the variance inflation factor, with a factor greater than 20 suggestive of multicollinearity.

\section{Results}

Between 2001 and 2010, a total of 760,418 (weighted $\mathrm{n}=3,743,613$ ) asthma admissions were included in our study cohort. Overall, $66.6 \%$ were female and $41 \%$ were white race. After excluding the $9.1 \%$ missing seasonal admission data, the rate of admissions was higher during winter and fall (26.2 and $25.2 \%$, respectively). Notably, most of the admissions were in the groups aged 35-54 and 55-74 years (31.3 and 26.3\%, respectively). Medicare/Medicaid was the primary payer $(57.4 \%)$ with only $11.8 \%$ of the subjects having private insurance. The majority of the admissions were done in large (59\%), urban $(85 \%)$ or nonteaching $(56.1 \%)$ hospitals. Of all the admissions, $7.4 \%$ required intubation/mechanical ventilation (table 1), as discussed in detail below. There was a small increase in asthma hospitalizations over the study period. Although admissions for female patients decreased to some extent, females accounted for the majority of admissions over the years (fig. 1).

The overall in-hospital mortality was $0.97 \%$ and as high as $9.8 \%$ in patients requiring intubation/mechanical 
Table 1. Patient and admission characteristics and outcomes stratified by use of intubation/mechanical ventilation

\begin{tabular}{lrrr}
\hline Demographic variable & \multirow{2}{*}{ All } & \multicolumn{2}{c}{ Intubation/mechanical ventilation } \\
\cline { 3 - 4 } & & \multicolumn{1}{c}{ no value } & yes \\
\hline Total asthma exacerbation hospitalizations (unweighted), $\mathrm{n}$ & 760,418 & $704,488(92.6)$ & $55,930(7.4)$ \\
Total asthma exacerbation hospitalizations (weighted), $\mathrm{n}$ & $3,743,613$ & $3,468,100(92.6)$ & $275,513(7.4)$ \\
\hline
\end{tabular}

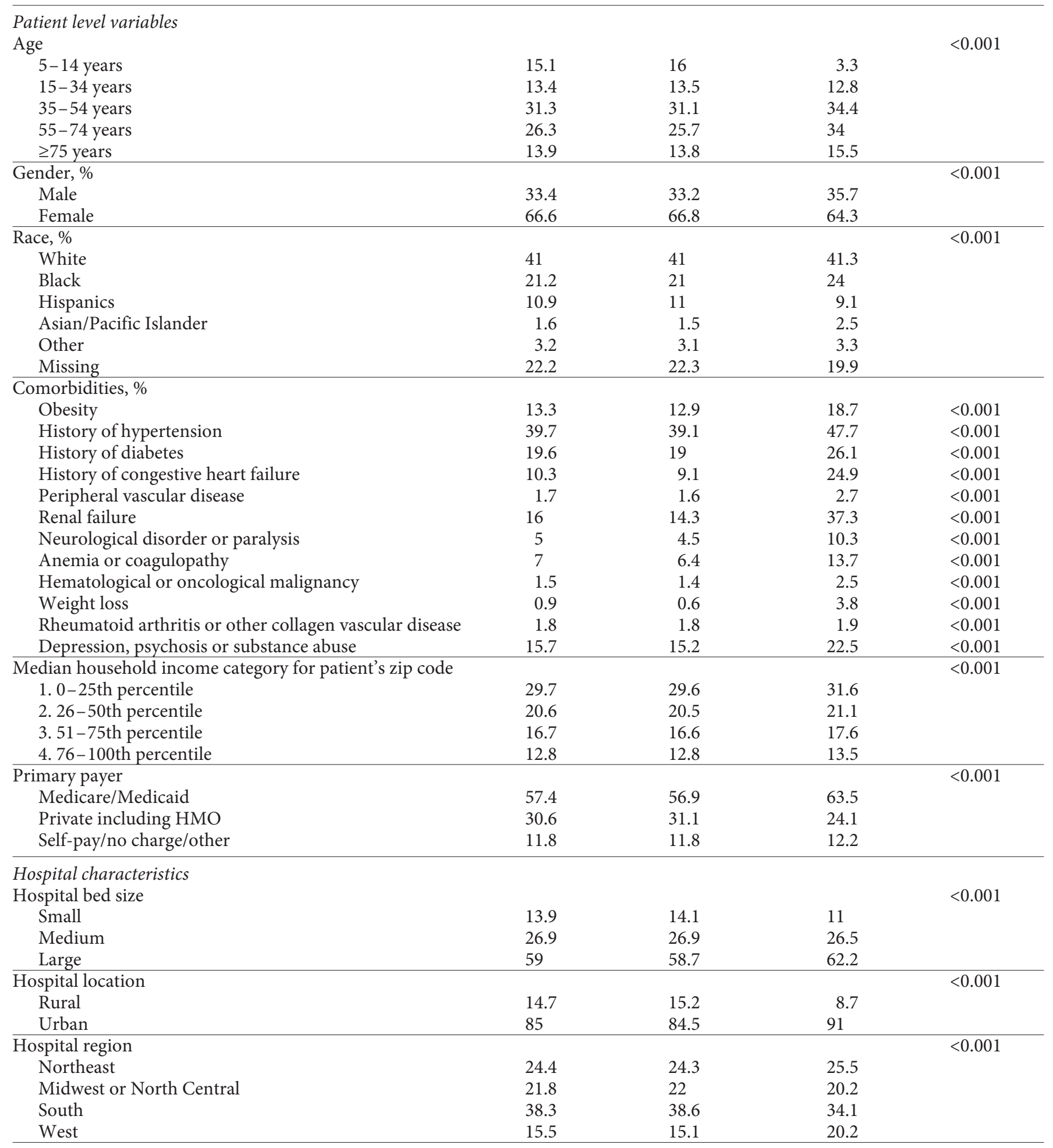

Asthma: In-Hospital Mortality and Hospitalization Costs in the USA
Int Arch Allergy Immunol 2015;168:71-78 DOI: $10.1159 / 000441687$ 
Table 1 (continued)

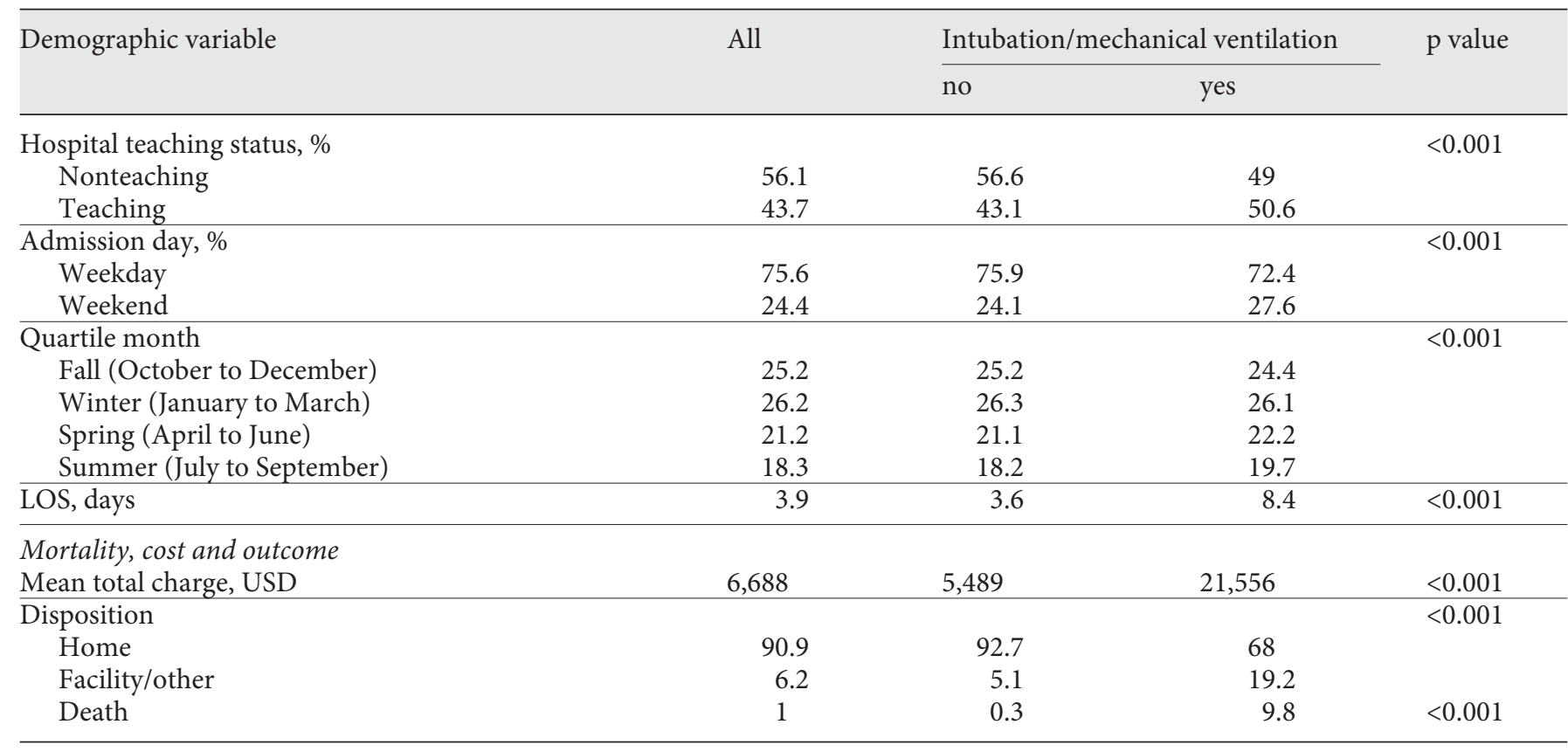

Fig. 1. Admission prevalence by gender and overall $(\mathrm{p}<0.001)$.

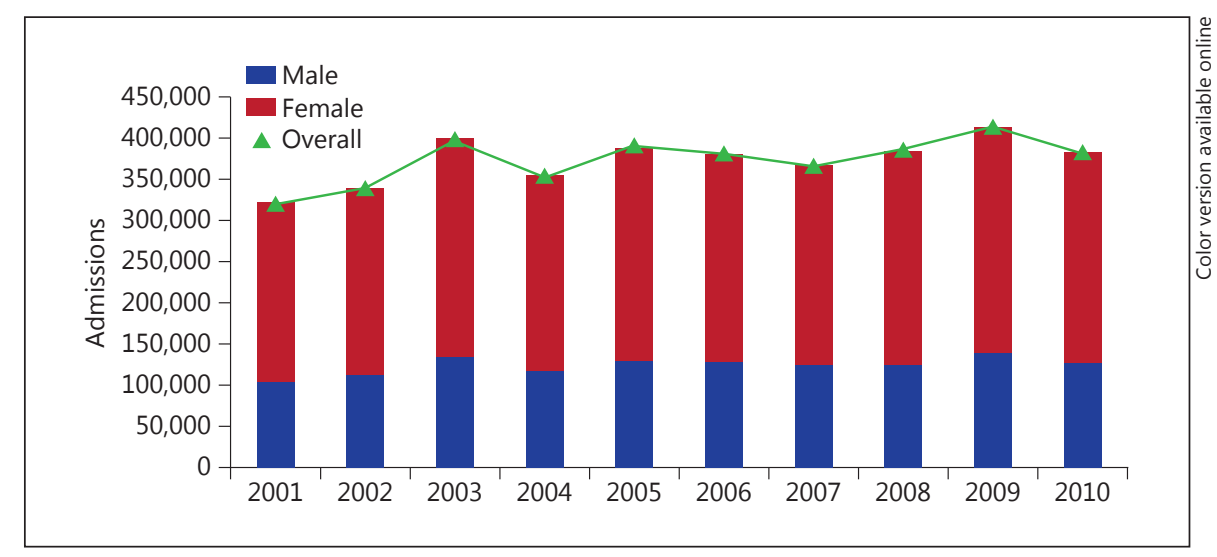

ventilation. The in-hospital mortality increased until 2006 and then remained relatively stable after a slight decrease (from $0.85 \%$ in 2001 to $0.97 \%$ in 2010 ; fig. 2). Private insurance was predictive of lower in-hospital mortality (odds ratio $0.72,95 \%$ CI $0.66-0.79, \mathrm{p}<0.001$ ). Other multivariate predictors of higher in-hospital mortality included increasing age, weekend admission and hospitalizations during the winter months, as well as Asian race (table 2).

The overall mean LOS was 3.9 days, and was as high as 8.3 days in patients requiring intubation/mechanical ventilation. After initially increasing, the average LOS decreased slightly, although it continued to be higher than in 2001 (from 3.7 days in 2001 to 3.9 days in 2010; fig. 3). Private insurance was predictive of a decreased LOS $(-0.47$ days, $95 \%$ CI -0.50 to $-0.45, \mathrm{p}<0.001)$. The multivariate predictors of a higher LOS included increasing age and hospitalization during the winter months (table 3).

The overall hospitalization cost was USD 6,688, and was as high as USD 21,556 in patients requiring intubation/mechanical ventilation. Over the years the hospitalization cost showed a steadily increasing trend (from USD 5,611 in 2001 to USD 7,230 in 2010; fig. 3). Private insurance was predictive of a decreased hospitalization cost (USD $-933,95 \%$ CI -997 to $-868, \mathrm{p}<0.001$ ). The 
Table 2. Univariate and multivariate analysis of in-hospital mortality

\begin{tabular}{|c|c|c|c|}
\hline & \multicolumn{2}{|c|}{ Univariate analysis } & \multirow{2}{*}{$\begin{array}{l}\text { Multivariate analysis } \\
\text { Relative risk } \\
\text { OR (UL-LL, p value) }\end{array}$} \\
\hline & $\begin{array}{l}\% \text { of } \\
\text { deaths }\end{array}$ & $\begin{array}{l}\text { Relative risk } \\
\text { OR (UL-LL, p value) }\end{array}$ & \\
\hline \multicolumn{4}{|l|}{ Race } \\
\hline White & 1.24 & Reference & Reference \\
\hline Black & 0.67 & $0.53(0.52-0.55,<0.001)$ & $0.90(0.81-1.00,0.05)$ \\
\hline Hispanics & 0.66 & $0.53(0.51-0.55,<0.001)$ & $0.66(0.57-0.77,<0.001)$ \\
\hline Asian/Pacific Islander & 2.14 & $1.74(1.64-1.84,<0.001)$ & $1.50(1.22-1.84,<0.001)$ \\
\hline Others & 0.9 & $0.72(0.68-0.77,<0.001)$ & $0.89(0.72-1.09,0.26)$ \\
\hline Missing & 0.86 & $0.69(0.67-0.71,<0.001)$ & $0.91(0.83-1.00,0.06)$ \\
\hline \multicolumn{4}{|c|}{${ }^{2}$} \\
\hline $5-14$ years & 0.08 & Reference & Reference \\
\hline $15-34$ years & 0.26 & $3.07(2.77-3.42,<0.001)$ & $3.54(2.54-4.93,<0.001)$ \\
\hline $35-54$ years & 0.49 & $5.92(5.39-6.50,<0.001)$ & $6.68(4.96-9.00,<0.001)$ \\
\hline $55-74$ years & 1.35 & $16.24(14.82-17.80,<0.001)$ & $16.56(12.35-22.22,<0.001)$ \\
\hline$\geq 75$ years & 3.02 & $37.11(33.87-40.66,<0.001)$ & $33.65(24.96-45.35,<0.001)$ \\
\hline \multicolumn{4}{|l|}{ Gender } \\
\hline Male & 1 & Reference & Reference \\
\hline Female & 0.97 & $0.96(0.95-0.99,0.003)$ & $0.74(0.69-0.79,<0.001)$ \\
\hline \multicolumn{4}{|c|}{ Median household income category for patient's zip code } \\
\hline 1. $0-25$ th percentile & 0.86 & Reference & Reference \\
\hline 2. 26-50th percentile & 1.07 & $1.23(1.20-1.27,<0.001)$ & $1.13(1.04-1.22,0.005)$ \\
\hline 3. $51-75$ th percentile & 1.04 & $1.2(1.20-1.24,<0.001)$ & $1.07(0.97-1.17,0.17)$ \\
\hline 4. $76-100$ th percentile & 1.2 & $1.39(1.35-1.44,<0.001)$ & $1.06(0.95-1.19,0.28)$ \\
\hline \multicolumn{4}{|l|}{ Primary payer } \\
\hline Medicare/Medicaid & 1.33 & Reference & Reference \\
\hline Private including HMO & 0.5 & $0.37(0.36-0.38,<0.001)$ & $0.72(0.66-0.79,<0.001)$ \\
\hline Self pay/no charge/other & 0.48 & $0.36(0.34-0.38,<0.001)$ & $0.77(0.67-0.88,<0.001)$ \\
\hline \multicolumn{4}{|l|}{ Admission day (\%) } \\
\hline Weekday & 0.96 & Reference & Reference \\
\hline Weekend & 1.03 & $1.07(1.05-1.10,<0.001)$ & $1.12(1.04-1.20,0.002)$ \\
\hline \multicolumn{4}{|l|}{ Quartile month } \\
\hline Fall (October to December) & 0.88 & Reference & Reference \\
\hline Winter (January to March) & 1.12 & $1.26(1.23-1.31,<0.001)$ & $1.13(1.04-1.22,0.003)$ \\
\hline Spring (April to June) & 0.97 & $1.1(1.07-1.14,<0.001)$ & $0.99(0.91-1.08,0.77)$ \\
\hline Summer (July to September) & 1 & $1(0.96-1.04,0.99)$ & $0.95(0.86-1.04,0.24)$ \\
\hline
\end{tabular}

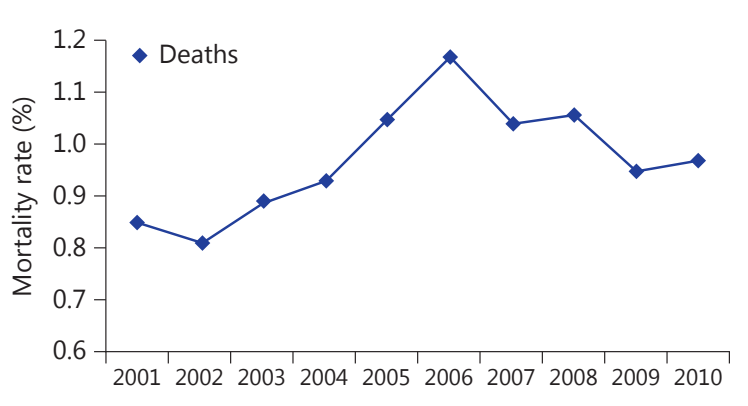

Fig. 2. In-hospital mortality $(\mathrm{p}<0.001)$.

Asthma: In-Hospital Mortality and Hospitalization Costs in the USA

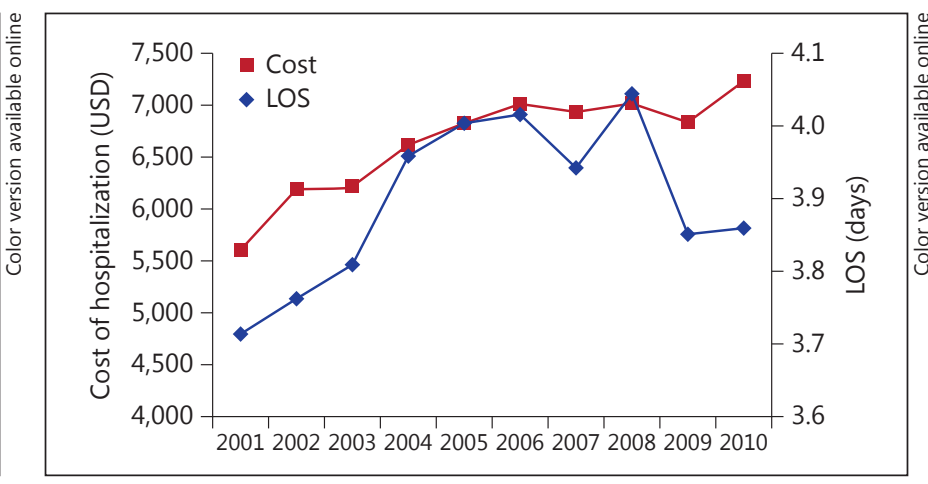

Fig. 3. LOS and hospitalization cost $(\mathrm{p}<0.001)$.

Int Arch Allergy Immunol 2015;168:71-78 DOI: $10.1159 / 000441687$ 
Table 3. Multivariate analysis of LOS and hospitalization cost

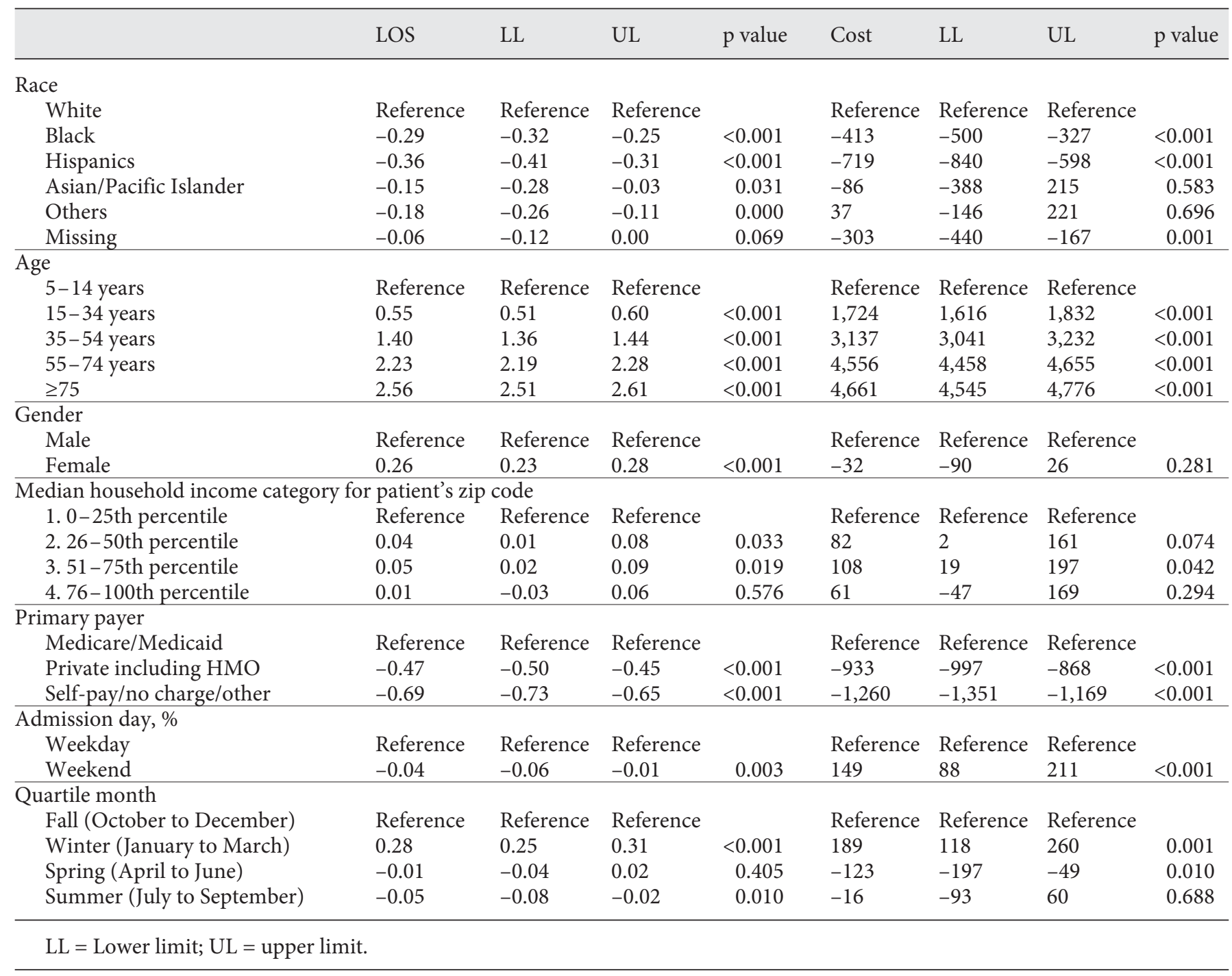

multivariate predictors of higher hospitalization cost included increasing age and hospitalization during the winter months (table 3).

Of all the admissions, $7.4 \%$ required intubation/mechanical ventilation. The rate of mechanical ventilation was higher during the winter (26.1\%) and fall (24.4\%) months. Also, the admissions requiring mechanical ventilation were associated with a higher resource utilization in terms of LOS (8.4 vs. 3.6 days, $\mathrm{p}<0.001$ ) and hospitalization cost (USD 21,556 vs. $5,489, \mathrm{p}<0.001$ ), a higher discharge to facility rate $(19.2$ vs. $5.1 \%, \mathrm{p}<0.001)$ and a higher death rate $(9.8$ vs. $0.27 \%, \mathrm{p}<0.001)$ as opposed to admissions that did not involve mechanical ventilation (table 1).

\section{Discussion}

Our study reports contemporary data for asthma hospitalizations in the USA over a 10-year period from the largest publicly available nationwide database. There was a slight increase in asthma hospitalizations over the last decade. Asthma continues to account for in-hospital mortality, especially in mechanically ventilated patients. Likewise, there was a significant increase in LOS and hospitalization costs in ventilated patients. Mechanically ventilated patients were older and had a higher burden of various baseline comorbidities, including hypertension, diabetes mellitus, renal failure, congestive heart failure, anemia and neurological disorders, etc. A higher propor- 
tion of mechanically ventilated patients were discharged to facilities. The overall rate of intubation in our study was higher than in previously reported data [10]. However, our study includes national data over several years with a larger sample size compared to previous studies [11]. One of the possibilities could be lack of quality and evidence-based measures to avoid intubation in acute asthma exacerbations at some participating hospitals, thus leading to higher intubation rates. However, the higher mortality rate in intubated patients observed in our study was similar to that in previously published literature [12]. Further studies with detailed hospitalization records, including the timing of and reason for intubation, might be valuable.

Similar to the previous literature [13], age was a significant predictor of in-hospital mortality in asthma patients in our study. A higher burden of underlying comorbidities in elderly patients could likely contribute to higher in-hospital mortality. Asthma hospitalization during the winter months was also significantly predictive of increased in-hospital mortality. This reaffirms previously reported data, wherein higher asthma-related mortality during the winter months was associated with a concurrently higher prevalence of respiratory viral infections, including influenza [14]. Furthermore, inhaling cold air has been known to trigger bronchoconstriction in asthmatics, which was also demonstrated by a previous observational study of cross-country skiers [15]. A prior study by Fleming et al. [16] also reported elderly patients to have a higher incidence of asthma exacerbations and asthma-related deaths during the midwinter months in England. Asians were more likely to die during an asthma exacerbation-related hospitalization in our study. Prior studies have reported higher hospitalization rates in Asians, likely secondary to poor medication compliance, language barriers and adherence to alternative medicine [17]. All these could potentially lead to a delay in seeking care and thus worse outcomes following hospitalization. Further studies would be needed to study racial differences in asthma exacerbations as well as their impact on in-hospital mortality.

There was a steady increase in asthma-related hospitalization costs over the last decade even after adjusting for inflation. Furthermore, our study results showed predictors of in-hospital mortality to also predict longer LOS and higher hospitalization costs. These included elderly patients and those that were admitted on weekends or during the winter months. Furthermore, patients with private insurance had lower in-hospital mortality coupled with lower LOS and hospitalization costs compared to Medicare/Medicaid patients. Previous studies have noted discrepancies in clinical outcomes between patients with private insurance and Medicare/Medicaid, with poorer outcomes noted for the latter $[18,19]$. Our study corroborated these observations for patients admitted with asthma exacerbations. The reasons for such disparity could include difficult access to health care, less acceptance by certain health systems, more comorbidities amongst Medicare/Medicaid patients besides some unobserved differences between payer types and outcomes. Private insurances are also reported to pay physicians and hospitals higher than public payers [20]. Further actionable strategies might be needed for a more equitable distribution of resources between all patients irrespective of the type of insurance.

Our study limitations are inherent to the analysis of a large administrative database, which could include underreporting of secondary and comorbid diagnoses, as well as coding errors. Furthermore, the observational nature of our study limits any causal inferences. We also lacked any information on the timing of intubation during hospitalization as well as the reason for discharge to facilities (e.g. myopathy, etc.). However, the NIS represents the largest publicly available database that has previously been widely used for research. It provided us with an opportunity to perform a comprehensive outcome analysis using a large sample size and a sound sampling design.

In summary, asthma continues to account for significant in-hospital mortality and resource utilization in terms of increasing hospitalization costs, especially in mechanically ventilated patients. Age, admissions during the winter months and the type of insurance are independent predictors of in-hospital outcomes. Future studies on promoting better asthma control in the outpatient setting, especially in high-risk populations, might aid in preventing hospitalization and associated in-hospital morbidity and mortality.

\section{Disclosure Statement}

The authors have no disclosures or conflicts of interest. No study-specific funding was used to support this work. The authors are solely responsible for the study design, conduct, and analyses and for drafting and editing of the article and its final contents. No statement should be construed as an official position of the Agency for Healthcare Research and Quality or the US Department of Health and Human Services.
Asthma: In-Hospital Mortality and

Hospitalization Costs in the USA
Int Arch Allergy Immunol 2015;168:71-78 DOI: $10.1159 / 000441687$ 


\section{References}

1 Akinbami LJ, Moorman JE, Bailey K, et al: Trends in asthma prevalence, health care use, and mortality in the United States, 20012010. NCHS data brief 94. 2012. http://www. cdc.gov/nchs/data/databriefs/db94.htm.

2 Centers for Disease Control and Prevention: Asthma: data, statistics and surveillance asthma surveillance data. http://www.cdc. gov/asthma/asthmadata.htm.

3 Akinbami LJ, Moorman JE, Liu X: Asthma prevalence, health care use, and mortality: United States, 2005-2009. National Health Statistics Reports 32. 2011. http://www.cdc. gov/nchs/data/nhsr/nhsr032.pdf.

4 Krishnan V, Diette GB, Rand CS, et al: Mortality in patients hospitalized for asthma exacerbations in the United States. Am J Respir Crit Care Med 2006;174:633-638.

5 Healthcare Cost and Utilization Project: Trend weights for HCUP NIS data. http:// www.hcup-us.ahrq.gov/db/nation/nis/trendwghts.jsp.

6 Steiner C, Elixhauser A, Schnaier J: The healthcare cost and utilization project: an overview. Eff Clin Pract 2002;5:143-151.
7 Epstein AJ, Polsky D, Yang F, Yang L, Groeneveld PW: Coronary revascularization trends in the United States, 2001-2008. JAMA 2011;305:1769-1776.

8 Healthcare Cost and Utilization Project: Overview of the National (Nationwide) Inpatient Sample (NIS). http://www.hcup-us. ahrq.gov/nisoverview.jsp.

9 US inflation calculator. http://www.usinflationcalculator.com.

10 Pendergraft TB, Stanford RH, Beasley R, et al: Rates and characteristics of intensive care unit admissions and intubations among asthmarelated hospitalizations. Ann Allergy Asthma Immunol 2004;93:29-35.

11 Zimmerman JL, Dellinger RP, Shah AN, Taylor RW: Endotracheal intubation and mechanical ventilation in severe asthma. Crit Care Med 1993;21:1727-1730.

12 Brenner B, Corbridge T, Kazzi A: Intubation and mechanical ventilation of the asthmatic patient in respiratory failure. J Allergy Clin Immunol 2009;124(suppl):S19-S28.

13 Boulet LP: Is asthma control really more difficult to achieve in the elderly patient? Int Arch Allergy Immunol 2014;165:149-151.

14 McCoy L, Redelings M, Sorvillo F, Simon P: A multiple cause-of-death analysis of asthma mortality in the United States, 1990-2001. J Asthma 2005;42:757-763.
15 Larsson K, Ohlsen P, Larsson L, et al: High prevalence of asthma in cross country skiers. BMJ 1993;307:1326-1329.

16 Fleming DM, Cross KW, Sunderland R, Ross AM: Comparison of the seasonal patterns of asthma identified in general practitioner episodes, hospital admissions, and deaths. Tho$\operatorname{rax} 2000 ; 55: 662-665$.

17 Hussein S, Partridge M: Perceptions of asthma in South Asians and their views on educational materials and self-management plans: a qualitative study. Patient Educ Couns 2002; 48:189-194.

18 Spencer CS, Roberts ET, Gaskin DJ: Differences in the rates of patient safety events by payer: implications for providers and policymakers. Med Care 2015;53:524-529.

19 Shi R, Taylor H, McLarty J, et al: Effects of payer status on breast cancer survival: a retrospective study. BMC Cancer 2015;15:211.

20 Medicare Payment Advisory Commission: Report to the Congress: Medicare payment policy. 2014. http://medpac.gov/documents/ reports/mar14_entirereport.pdf. 